\title{
On the Electron Extraction in a large RF Driven Negative Hydrogen Ion Source for the ITER NBI System
}

\author{
P. Franzen, D. Wünderlich, U. Fantz and NNBI Team \\ Max-Planck-Institut für Plasmaphysik, EURATOM Association, Boltzmannstraße 2, \\ 85748 Garching, Germany \\ E-mail contact of main author: peter.franzen@ipp.mpg.de
}

\begin{abstract}
The test facility ELISE, equipped with a large RF driven ion source $\left(1 \times 0.9 \mathrm{~m}^{2}\right)$ of half the size of the ion source for the ITER neutral beam injection (NBI) system, was constructed in the last three years at the Max-Planck-Institut für Plasmaphysik (IPP), Garching, and is now operational. First measurements of the dependence of the co-extracted electron currents on various operational parameters have been performed. ELISE has the unique feature, that the electron currents can be measured individually on both extraction grid segments, leading to some vertical spatial resolution. Although done in volume operation, where the negative hydrogen ions are created in the plasma volume solely, the results are very encouraging for the operation with cesium, the latter being necessary in order to achieve the relevant negative ions currents for the ITER NBI injectors. The amount of co-extracted electrons could be suppressed sufficiently with moderate magnetic filter fields and by plasma grid bias. Furthermore, the electron extraction is more or less decoupled from the main plasma, as the observed vertical asymmetry of electron extraction is not correlated at all with the plasma asymmetry, which is anyway rather small. Both effects are superior to the experience from the small IPP prototype source; the reason for these encouraging results are most probably the larger size of the source as well as the new geometry of the source having unbiased areas in the centre of the source. The reasons, however, for the observed asymmetry of the extracted electron currents and their dependencies on various operational parameters are not well understood.
\end{abstract}

\section{Introduction}

For heating and current drive the ITER NBI system [1,2] requires a negative hydrogen ion source capable of delivering up to $57 \mathrm{~A}^{-}$of $\mathrm{D}^{-}$ions for up to one hour. In order to achieve the required $40 \mathrm{~A}$ of accelerated current at $1 \mathrm{MeV}$ - corresponding to an accelerated current density of $200 \mathrm{~A} / \mathrm{m}^{2}$ - and to limit the power loads in the extraction system, the ion losses in the accelerator must be minimized by operating the source at a pressure of $0.3 \mathrm{~Pa}$ and the amount of co-extracted electrons must not exceed the amount of extracted negative ions. Presently these parameters have not yet been achieved simultaneously, partly due to a lack of adequate test facilities. Thus the European ITER domestic agency F4E has defined an R\&D roadmap for the construction of the neutral beam heating systems $[3,4]$. An important step herein is the new test facility ELISE (Extraction from a Large lon Source Experiment) for a large-scale extraction from a half-size ITER RF source ( $1 \times 0.9 \mathrm{~m}^{2}$ with an extraction area of $0.1 \mathrm{~m}^{2}$ ) which was constructed in the last years at Max-Planck-Institut für 
Plasmaphysik (IPP) and is now operational [5,6]. The early experience of the operation of such a large RF driven source will give an important input for the design of the Neutral Beam Test Facility PRIMA in Padova [7] and the ITER NBI systems (heating beams and diagnostic beam [8,9], the latter being built by Indian ITER Domestic Agency) and for their commissioning and operating phases. PRIMA consists of the $1 \mathrm{MeV}$ full power test facility MITICA [10], operational in 2017, and the $100 \mathrm{kV}$ ion source test facility SPIDER [11], operational in 2015.

One of the most critical issues for fusion relevant negative hydrogen ion sources is the suppression of the electrons which are inevitably co-extracted with the negative hydrogen ions $[12,13,14,15]$. These electrons have to be filtered out of the beam before being accelerated to high energies; this is done by deflecting the electrons to the so-called extraction grid (see below) by the magnetic field created by magnets embedded inside the grid. In order to keep the electron power below a technically feasible limit, the voltage in this first extraction step has to be rather low. Nevertheless, the resulting power densities are still very high (up to $40 \mathrm{MW} / \mathrm{m}^{2}$ for the ITER relevant electron currents); as due to the complex magnetic field structure inside the extraction system, the electrons are focused on very localised spots of roughly $1 \mathrm{~mm}^{2}$ [16]. Several countermeasures have to be undertaken to limit the co-extracted electron current and its power and power density: (1) a sufficient low extraction voltage of $10 \mathrm{kV}$ at maximum, (2) biasing the first grid (the so-called plasma grid, PG) positively with respect to the source by few tens of $V$ [13], and (3) a magnetic filter field of the order of some $\mathrm{mT}$ in front of the plasma grid and an integrated magnetic field of about $1 \mathrm{mTm}$ through the source leading to a reduction of the electron temperature and density [17]. These measures, however, decrease also the extracted ion current, so that the power load capability of the extraction grid limits also accelerated current of the source. This power load limit of the extraction grid is especially a problem in deuterium operation, where the co-extracted electron current is much larger (factor $3-4$ ) than in hydrogen $[15,18,19]$ even when the lower required accelerated currents in the ITER NBI system (40 A Dcompared to $\left.46 \mathrm{~A} \mathrm{H}^{-}[1]\right)$ are taken into account.

This paper reports in detail the first experiments for a large RF driven ion source regarding the suppression of the amount of the co-extracted electrons. It discusses the various dependencies on the magnetic and electric fields in the source. Furthermore, one of the novel special features of ELISE is the fact that for the first time the electron current can be measured with some (low) spatial resolution, giving access to connect the asymmetries of the extracted electron currents which have been observed during the start-up phase [5] with the plasma asymmetries that might occur due to electron drifts in the magnetic filter field $[20,21]$.

In order to achieve the ITER relevant negative hydrogen ion currents, the use of the surface $\mathrm{H}^{-}$production process is presently mandatory: here the negative ions are produced 
at the plasma grid surface near the extraction apertures by a conversion of mainly neutral hydrogen atoms $[22,23]$. The necessary low work function of the plasma grid surface is achieved by evaporating cesium onto that surface $[12,13,14]$. The experiments reported here, however, have been performed in volume operation, i.e. without the use of cesium, where the negative hydrogen ions are generated by processes in the plasma volume leading to low ion currents $\left(20-30 \mathrm{~A} / \mathrm{m}^{2}\right.$, roughly an order of magnitude lower than required) and hence to large electron/ion ratios of the order of ten. But the results reported in this paper have some consequences for the cesium operation and can additionally be used as benchmark of large ion source models which are presently under development (see the overview in Ref. [24]).

\section{The ELISE Experiment}

Table 1 shows the main parameters of the ELISE test facility. The aim of the design of the ELISE source and extraction system was to be as close as possible to the ITER design $[13,25]$; it has however some modifications allowing a better diagnostic access as well as more flexibility for exploring open questions, as for example the suppression of the coextracted electrons reported in this paper. Plasma operation of up to one hour is foreseen; but due to the technical limits of the IPP HV system, pulsed extraction only is possible.

The ELISE test facility is equipped with a half-size ITER RF source $\left(1 \times 0.9 \mathrm{~m}^{2}\right)$ with the same width but half the height of the ITER NBI source. Accordingly, the plasma is generated in four cylindrical drivers (instead of eight as for the ITER source) with a diameter of $300 \mathrm{~mm}$. The RF power is coupled inductively into the plasma via a six-turn copper coil wound around an $\mathrm{Al}_{2} \mathrm{O}_{3}$ insulator; the latter is protected from plasma sputtering by an actively cooled copper Faraday screen. ELISE is equipped with two $180 \mathrm{~kW}$ RF generators, each of which drives a pair of two horizontal drivers in series, as it is the case for the ITER NBI sources. The matching of the load to the generator is done by a combination of a series and a parallel

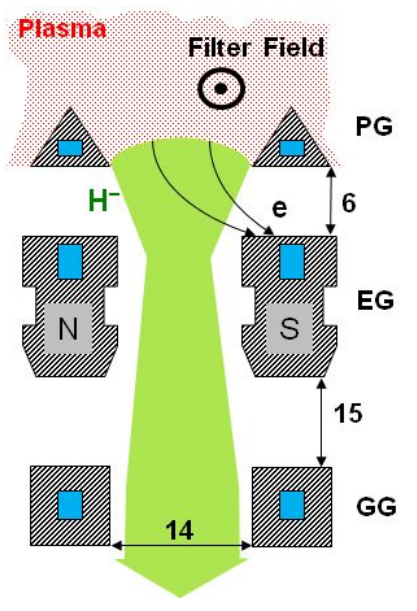

Figure 1: Sketch of the extraction system of ELISE also indicating the magnetic fields. Dimensions are given in $\mathrm{mm}$. 
capacitor, the latter being remotely tuneable for a possible change of the matching during a pulse.

The ELISE extraction system is designed for acceleration of negative hydrogen ions of up to $60 \mathrm{kV}$. It consists of three grids (see Figure 1): the plasma grid (PG) which separates the plasma from the beam region, the extraction grid (EG), where the coextracted electrons are filtered out of the beam by the fields from permanent magnets embedded in the extraction grid, and the grounded grid (GG). Each grid has 640 extraction

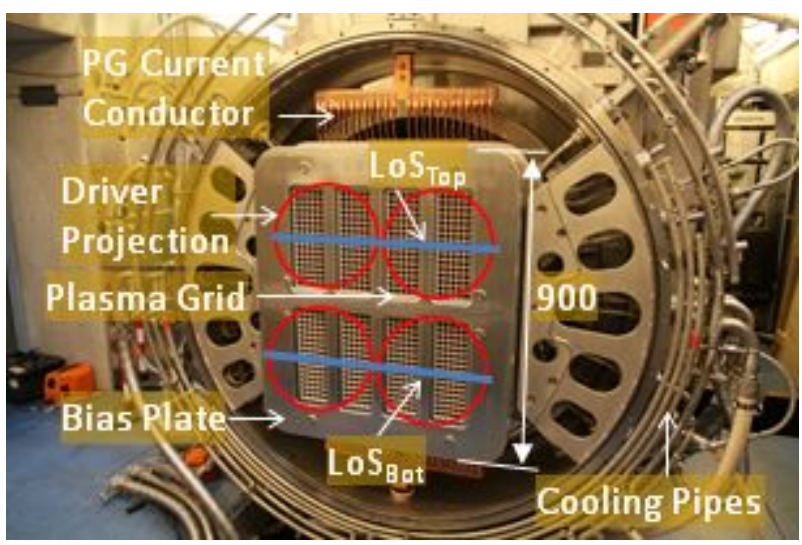

Figure 2: View onto the grid assembly of ELISE with the bias plate and plasma grid. Also indicated are the projections of the drivers and the Lines-of-Sight (LoS) at the top and the bottom drivers for the plasma asymmetry measurements with optical emission spectroscopy. Dimensions are given in $\mathrm{mm}$. apertures with a diameter of $14 \mathrm{~mm}$ (PG and GG) and $11 \mathrm{~mm}$ (EG), respectively, and consists of two segments in a vertical arrangement, i.e. a top and a bottom segment. The apertures are arranged in eight groups of $5 \times 16$ apertures (see Figure 2), which have to be aligned at each grid within $\pm 0.2 \mathrm{~mm}$ to ensure good beam optics. In order to suppress the amount of co-extracted electrons, the plasma grid is positively biased with respect to the source. A socalled bias plate (see Figure 2) extends the surface at source potential close to the edge of each aperture group of the plasma grid, so decreasing the area at the PG potential 'seen' by the plasma. From the experience with the small IPP prototype source, this bias plate is mandatory for the suppression of the co-extracted electrons [26], but in contrast to the small IPP prototype source the bias plate of the ELISE source surrounds the apertures not only at the edges of the source but also between aperture groups both horizontally and vertically.

Each of the segments of the ELISE extraction grid has two individual cooling circuits (see Figure 3). This is in contrast to the ITER $\mathrm{NBI}$ extraction grid having only one cooling circuit due to the limited space there; the different ELISE design was done to enhance the power load capability of the extraction grid $[16,25]$ for more experimental flexibility with respect to electron extraction. Furthermore, both EG segments are insulated against each other and against their grid holder boxes, so that the current flowing from each grid can be measured

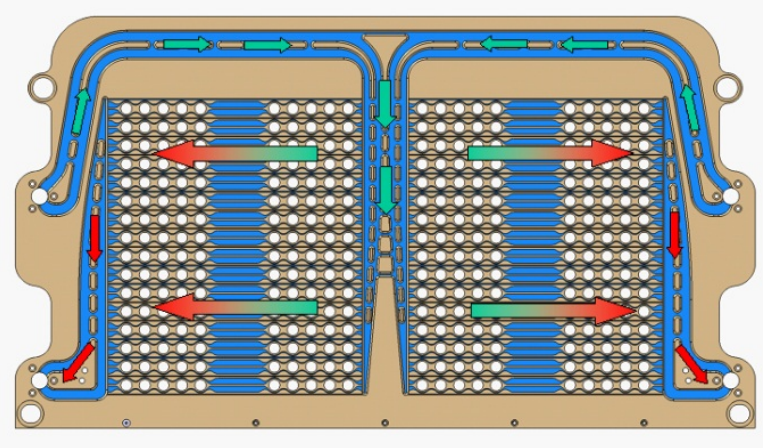

Figure 3: CAD drawing of one of the EG segments indicating the two individual cooling channels. 
individually. This gives the unique opportunity to investigate possible asymmetries also in electron extraction which is done in a first step in this paper - in order to enhance the understanding of the electron extraction processes in the ion source.

Figure 4 shows a

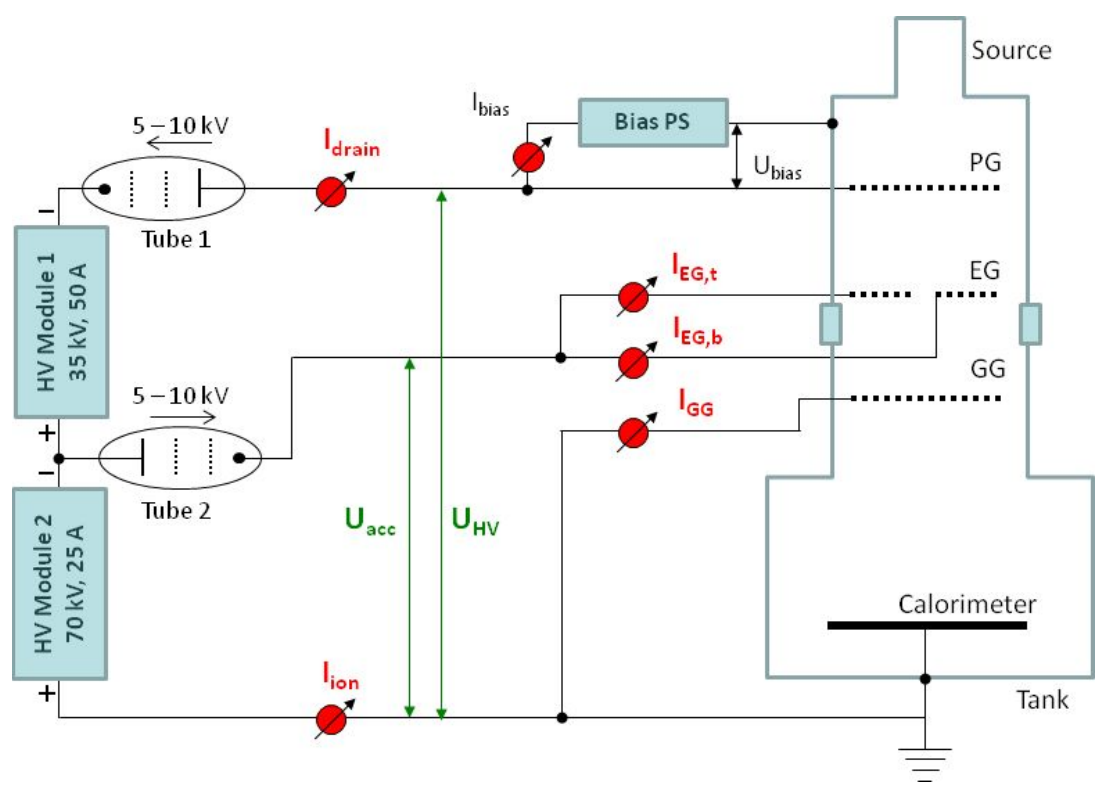
schematic overview of the Figure 4: Scheme of the HV circuit of ELISE with the voltage and HV circuit of ELISE. The current measurements. The voltage is regulated by tetrodes. ions are accelerated to a calorimeter located at a distance about $3.5 \mathrm{~m}$ from the GG. The source is at a high negative potential with a maximum voltage of $60 \mathrm{kV}$; regulated are the total voltage $\left(\mathrm{U}_{\mathrm{HV}}\right)$ and the acceleration voltage $\left(U_{\text {acc }}\right)$ between the extraction and the ground grid. The extraction voltage $\left(U_{e x}\right)$ is then just the difference between those two. The electrical currents flowing onto the grids, as well as the current flowing back to the HV power supply, are measured individually. The current on the extraction grid and on the grounded grid, respectively, can be caused in principle both by electrons and by negative ions; there are however indications (see below) that for the measurements reported here the current on the extraction grid can reasonably well be assumed to be solely caused by impinging electrons and the current on the grounded grid and on the beam line components (calorimeter) to be solely caused by negative hydrogen ions. This is in agreement also with the findings for the small IPP prototype source [12]. Hence, the ' $\mathrm{IEG}_{\mathrm{EG}}$ current measurements gives the current of electrons hitting the extraction grid segments, i.e. the 'co-extracted electron current' and the 'I inn $^{\prime}$ current measurement gives the current of negative hydrogen ions being extracted from the plasma grid, i.e. the 'extracted ion current'.

The current accountability is quite good: the sum of the currents flowing back to the HV power supplies is within $\pm 5 \%$ of the drain current [5]. Furthermore, the power onto the extraction grid during a pulse measured by calorimetry agrees also within $\pm 10 \%$ with the power calculated from the measured current and the extraction voltage [6]. The cooling water calorimetry gives also the opportunity to measure also left/right asymmetries; but these are small [6]. In the following, only the top/bottom asymmetry is discussed, as the magnetic filter field, the main driver of plasma asymmetries, has mainly horizontal components. 
The magnetic filter field in the RF driven cesiated negative hydrogen ion source is by far not optimized with respect to the strength, position, gradients, etc. for maximum negative ion yield and electron suppression. The ITER requirements have been obtained in the small IPP prototype source $\left(0.5 \times 0.3 \mathrm{~m}^{2}\right)$ with the magnetic filter field created by permanent magnets $[12,14,17,27]$. How these results can be transferred to the large ITER sources like the one for ELISE where the field is created by a current running through the plasma grid (see Ref. [28] and Figure 2) is one of the main issues of the ELISE experiments. Figure 5 shows as an example a comparison of the filter fields from the plasma grid to the

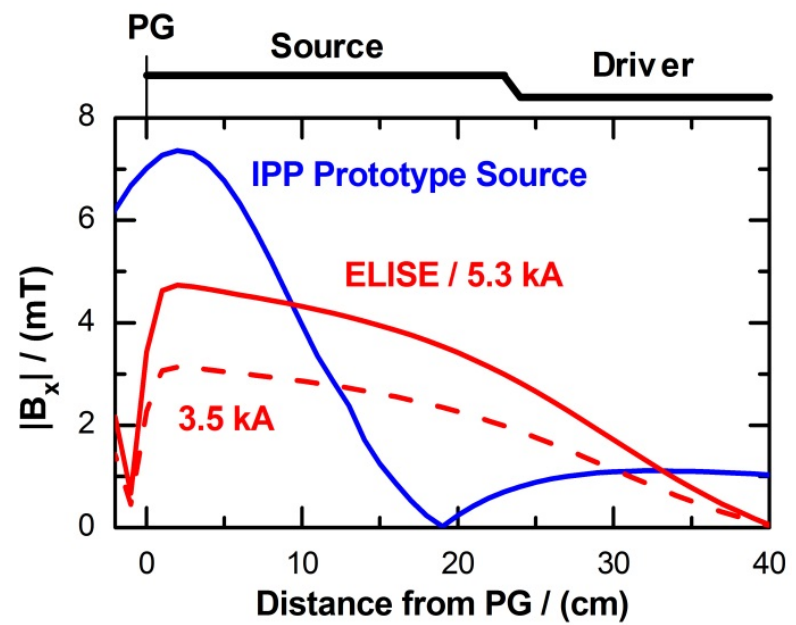

Figure 5: Comparison of the horizontal magnetic filter field in the centre of the source from the plasma grid (PG) to the driver for the small IPP prototype source (permanent magnets) and the larger ELISE source for $5.3 k A$ and $3.5 k A$, respectively. driver in the centre of the source for ELISE and the small IPP prototype source, clearly indicating the different field gradients. The filter field gradients differ also in the direction parallel to the plasma grid: the field generated by permanent magnets increases strongly when approaching the magnets - leading to a magnetic mirror for the electrons; this is not the case when the filter field is generated by the PG current. The integrated filter field, however, from the exit of the driver to the plasma grid - found to be the important parameter for electron suppression for the small IPP source [17] - is similar for both sources as this was the design value for the ELISE magnetic filter field (around $1 \mathrm{mTm}$, see Ref. [28] and [29]; in the latter the ELISE filter fields are shown for a different configuration of the current return conductors). Also shown in Ref. [28] and [29] is that the filter field structure parallel to the ELISE plasma grid shows some ripple due to the fact that the PG current can only flow in between the apertures.

The presently installed power supply can deliver $5.3 \mathrm{kA}$ at maximum, but due to a damage of one of the modules, only 3.5 kA have been available for the present studies. The current path in the plasma grid and the remaining magnetic filter field direction is presently so that - from the experience with the small prototype source - a possible ExB drift of the electrons on the plasma side should be upwards. In contrast to the filter field created with permanent magnets - as it is the case at the small prototype source - the field direction is reversed at the downstream side of the plasma grid with respect to the upstream side, so that negative charges are deflected downwards in the extraction system, as seen on the calorimeter (not shown here). The electron deflection field - created by the deflection magnets in the extraction grid and having also field components upstream the plasma grid 
with a strength comparable to the filter field - does not contribute to vertical asymmetries: in the present configuration both fields, i.e. electron deflection field and filter field are orthogonal to each other, so that the electron are deflected horizontally to the extraction grid by this electron deflection field (see Figure 1 and Ref. [25]). Furthermore, the field direction is reversed from aperture row to aperture row.

In the following, the asymmetry of parameters measured at the top and the bottom part of the source, respectively, is defined by

$$
A=\frac{y_{t}-y_{b}}{y_{t}+y_{b}}
$$

with $y_{t}$ and $y_{b}$ being the respective measurements at top and bottom. With that definition, a symmetric distribution is given by $A=0$, whereas $A= \pm 1$ indicates full asymmetry, i.e. almost no signal in the upper part $(A=-1)$ or in the lower part $(A=+1)$ of the source, respectively.

For the plasma asymmetry, the $\mathrm{H}_{\beta}$ emissivity at the two horizontal channels central to the drivers are taken (see Figure 2; for the details of the optical emission spectroscopy system at ELISE see Ref. [30]) as the $\mathrm{H}_{\alpha}$ line is prone to overexposure in the spectrometer. The center of both lines-of-sight has a distance of $21 \mathrm{~mm}$ from the bias plate and $34 \mathrm{~mm}$ from the plasma grid surface, the diameter of the lines-of-sight is $10-15 \mathrm{~mm}$. The $\mathrm{H}_{\beta}$ emissivity is in principle proportional to the electron density, electron temperature and the density of atomic hydrogen. But for the sake of simplicity the emissivity is taken as a global indication of the plasma asymmetry, as the relative contribution of the different parameters to the emissivity for the plasma conditions reported in this paper is still under evaluation.

In the following, the dependence of the currents on the upper and lower segment of the extraction grid and of their asymmetry on various operational parameters in hydrogen is discussed. The results are then compared with the asymmetry of the plasma in the above given distance from the plasma grid and the bias plate. The measurements have been performed in two different operational scenarios: firstly, the standard operation with both pairs of drivers being powered by the two generators, and secondly, an operation where only the upper or the lower pair of drivers has been powered. The latter one was done in order to investigate the crosstalk between the plasma generated in one half of the ion source with the EG segment in the other half.

\section{Results}

\subsection{Electron Suppression}

Figure 6 shows for the standard operation in hydrogen volume operation the basic dependencies of the extracted electron currents and of the negative ion current for the main parameters they are known to depend on, i.e. extraction voltage, PG bias and magnetic filter field. The amount of co-extracted electrons and negative ions increases with increasing 
extraction voltage, but the electron currents decrease with increasing magnetic filter field, i.e. plasma grid current, and with increasing plasma grid bias current. In contrast, the extracted ion current increases with increasing filter field and bias current. For these experiments, the bias circuit was operated in current control mode instead of voltage control; this mode has been found to be beneficial for cesiated negative hydrogen ion sources, as so the fluxes to the plasma grid are kept constant $[12,31]$.

The parameters for these scans are determined by the extraction grid power limit, presently set to $75 \mathrm{~kW}$. Therefore the extraction voltage must be rather low in order to have a wide parameter range of bias currents or filter field currents; correspondingly large variation of the extraction voltage is only possible for sufficient large values of the bias current or the filter field current, respectively.

These first experiments at ELISE

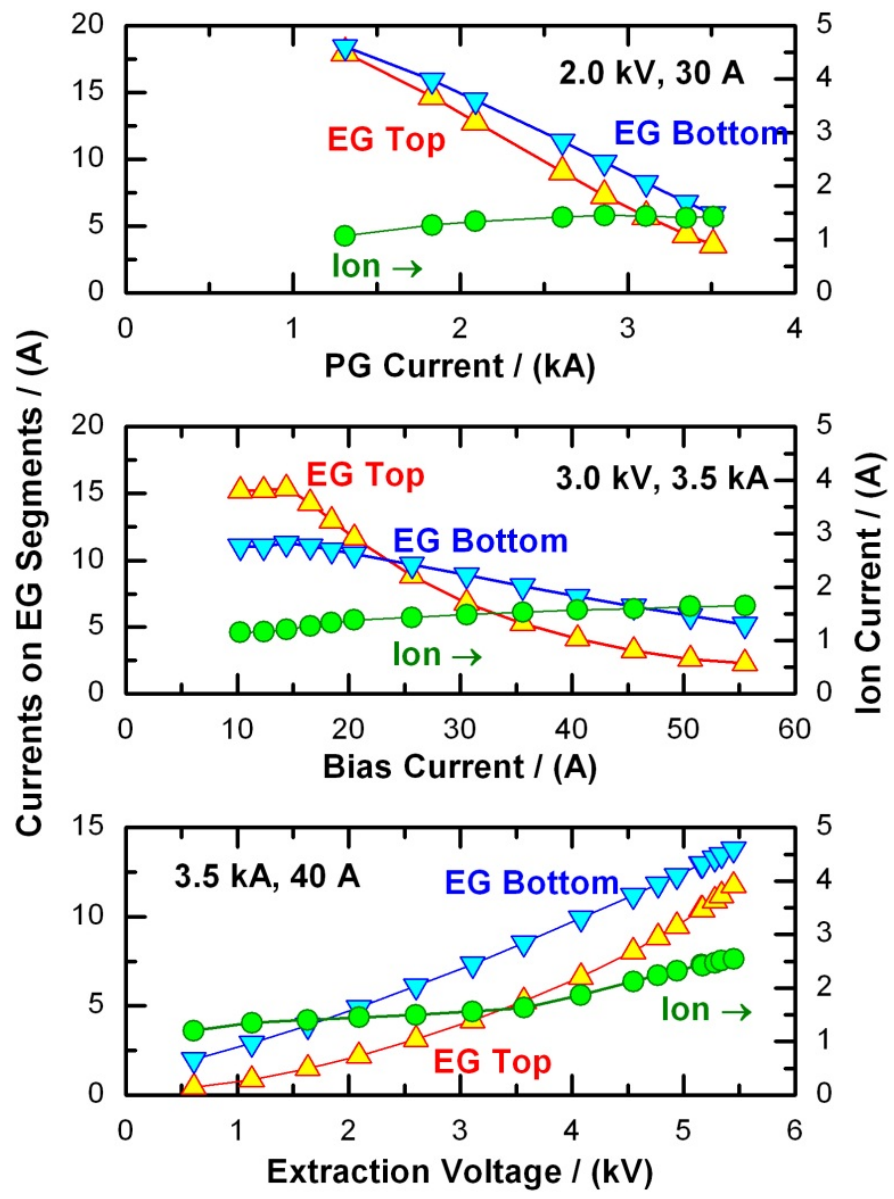

Figure 6: Dependence of the extracted currents (EG segments top and bottom, ion current) on the magnetic filter field current (top) $P G$ bias current (middle) and extraction voltage (bottom), in volume operation, i.e. w/o Cs. RF power was $2 \times 40 \mathrm{~kW}$ and the source filling pressure 0.6 Pa.

show that the electron current can be suppressed reasonably well with the magnetic filter field even in volume operation. An increase of the PG current from 1.5 to $3.5 \mathrm{kA}$ decreases the amount of co-extracted electrons by a factor of about three, an increase of the bias current from 10 to $55 \mathrm{~A}$ - corresponding to an increase of the bias voltage from 10 to $30 \mathrm{~V}$ - decreases the electron current by factors of two or seven for the given parameters.

This effective electron suppression is very encouraging also for the operation with Cs, larger RF power and deuterium, especially when taking the effect into account, that the plasma asymmetry as well as the plasma parameters change only slightly for these high filter field currents [30] and that these electron currents are already in the expected relevant range: for the required $20 \mathrm{~A}$ accelerated negative ion current which corresponds to the half of the required value for the ITER NBI, an extracted ion current of $25-30 \mathrm{~A}$ is necessary 
accounting for the different losses in the accelerator. This is also then the upper limit of the total electron current for the required electron/ion ratio of less than one.

The high ion currents reported here for the rather low applied RF power, i.e. the ion current of 2-3 A, corresponding to $20-30 \mathrm{~A} / \mathrm{m}^{2}$, are quite large for volume operation, and they indicate also a larger $\mathrm{H}^{-}$ production efficiency than the smaller sources: for a similar pressure $(0.6 \mathrm{~Pa})$ in volume operation, about $70 \mathrm{~kW}$ RF power per driver was necessary at BATMAN for the same

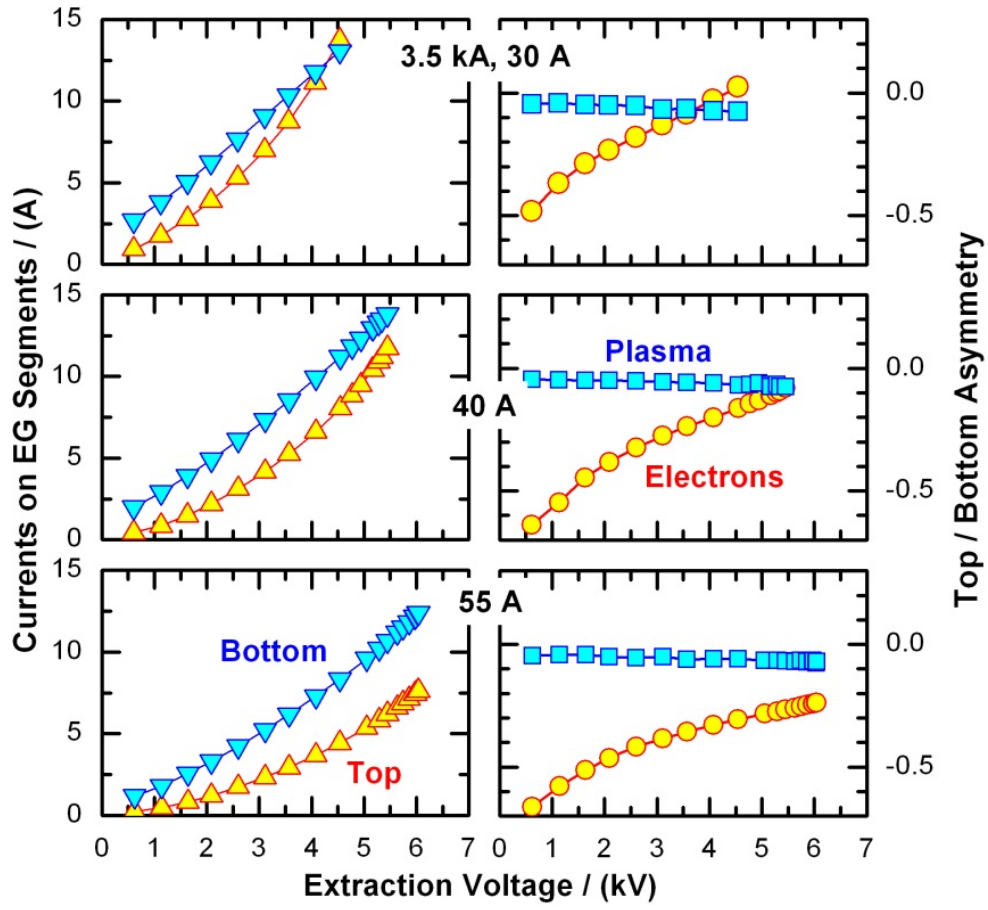

Figure 7: Dependence of the extracted currents on the extraction voltage in volume operation, i.e. W/o Cs. RF power was $2 \times 40 \mathrm{~kW}$; bias current $30 \mathrm{~A}, 40 \mathrm{~A}$ and $55 \mathrm{~A}$, source filling pressure 0.6 Pa, PG filter field current $3.5 \mathrm{kA}$. extracted ion current (see Ref.

[12], the quoted measured RF power there is too large by 30\%). Furthermore, the opposing trend of electron and ion currents with increasing plasma grid bias or magnetic filter field is an strong indication, that the measured current on the grounded grid is mostly composed by ions; additionally, the ion current is still so low, that even in the case of ion losses in the extraction grid, the current of the extraction grid is accordingly caused mostly by electrons.

Figure 6 shows also the aforementioned asymmetry of the electron currents measured at the upper and lower extraction grid segment. Their dependence on the plasma parameters and the comparison with the plasma asymmetry is discussed in detail in the next section.

\subsection{Asymmetry for Operation with Two Generators}

Figure 7 shows the dependence of the extracted electron currents on the extraction voltage for three different values of the bias current. The maximum extraction voltage in this parameter scan is again determined by the extraction grid power limit of $75 \mathrm{~kW}$; the higher the bias current, the lower the extracted electron currents (see next figure) and hence the higher the extraction voltage that can be achieved (presently $6 \mathrm{kV}$ for $55 \mathrm{~A}$ bias current and 3.5 kA PG current). The asymmetry of electron extraction decreases from about $-50 \%$ to almost zero with increasing extraction voltage; the amount of the decrease itself increases 
with increasing bias current. The plasma asymmetry, however, is rather low ( $<10 \%$ absolute) and changes only slightly with extraction voltage.

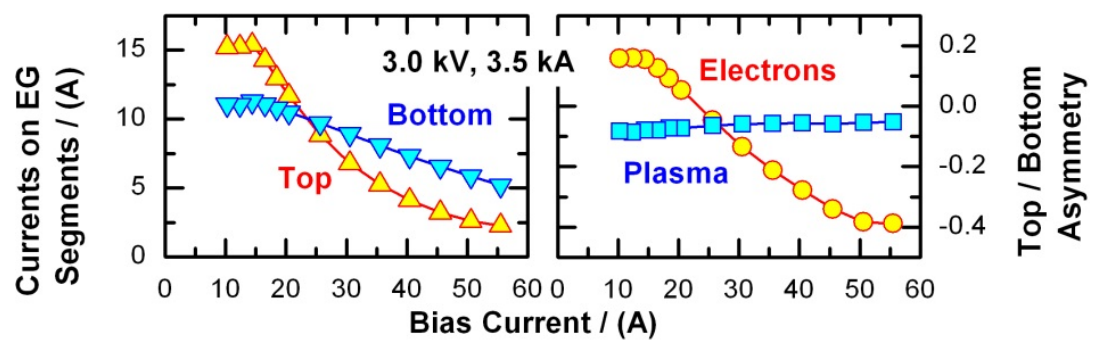

Figure 8 shows again Figure 8: Dependence of the extracted currents and their asymmetry in detail the dependence of the extracted electron currents on the bias filter field current $3.5 \mathrm{kA}$.

current and the comparison with the plasma asymmetry. As indicated implicitly in the last figure, the asymmetry of the electron extraction increases with bias current (and therefore also with the bias voltage). Interesting here is that the asymmetry changes the sign when the bias current is below $20 \mathrm{~A}$ : here more electrons are extracted from the top part of the source. Again, the plasma asymmetry is rather low and changes only slightly with the bias current.

Figure 9 shows the dependence of the extracted electron currents and their asymmetry on the plasma grid current, i.e. magnetic filter field. As mentioned before, a filter field current of $3.5 \mathrm{kA}$ was the maximum value due to a damaged power supply. Again the electron currents can be suppressed very effectively - by factors of five to ten - with increasing filter field; the asymmetry increases from almost zero to $-20 \%$ to $-40 \%$ with increasing filter field. As before, the amount of the increase depends on the bias current.

Also, the plasma asymmetry is quite low and changes only slightly with the PG current for the pulses where extraction was possible $(>1 \mathrm{kA})$ due to the EG power limit. Also shown here is the plasma asymmetry for pulses with low PG filter field current, where extraction was not possible, i.e. just in the RF phase. As discussed in detail in Ref. [30], the plasma, i.e. the plasma emission distribution, is much more sensitive to the filter field for PG currents below 1.5 kA. The small asymmetry of $10 \%$ in the case without magnetic filter field at all is most probably

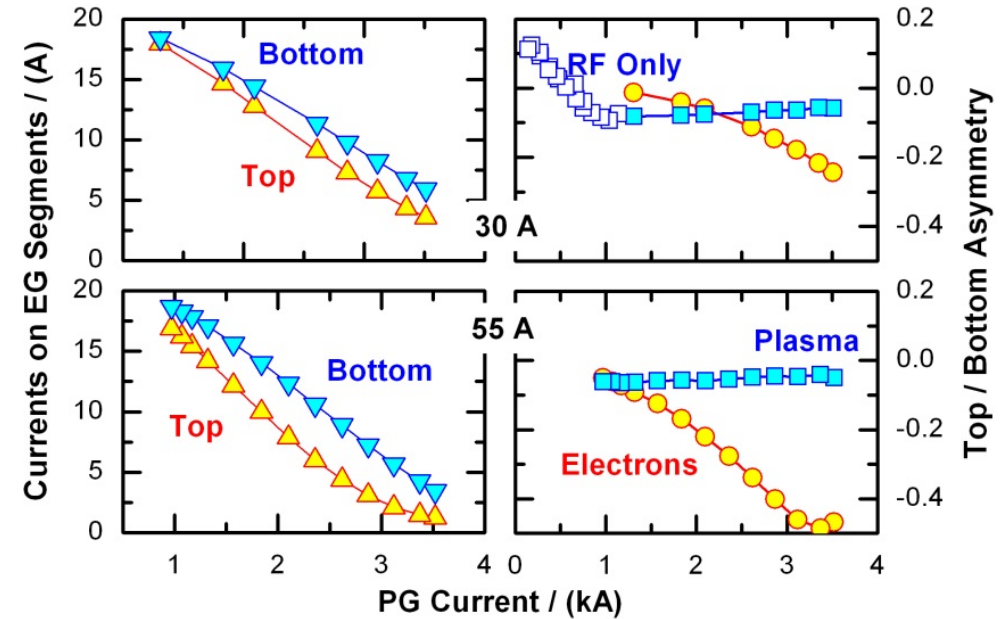

Figure 9: Dependence of the extracted currents and their asymmetry on the PG filter field current in volume operation, i.e. w/o Cs. RF power was $2 \times 40 \mathrm{~kW}$; bias current $30 \mathrm{~A}$ and $55 \mathrm{~A}$, source filling pressure $0.6 \mathrm{~Pa}$, extraction voltage $3 \mathrm{kV}$. 
a consequence of the different RF power control units for both generators: a small change of $3 \mathrm{~kW}$ only in one generator (less than $10 \%$ of the set power) lead to $A=0$. This $10 \%$ is also then the error in the plasma asymmetry. The rather constant and small plasma asymmetry

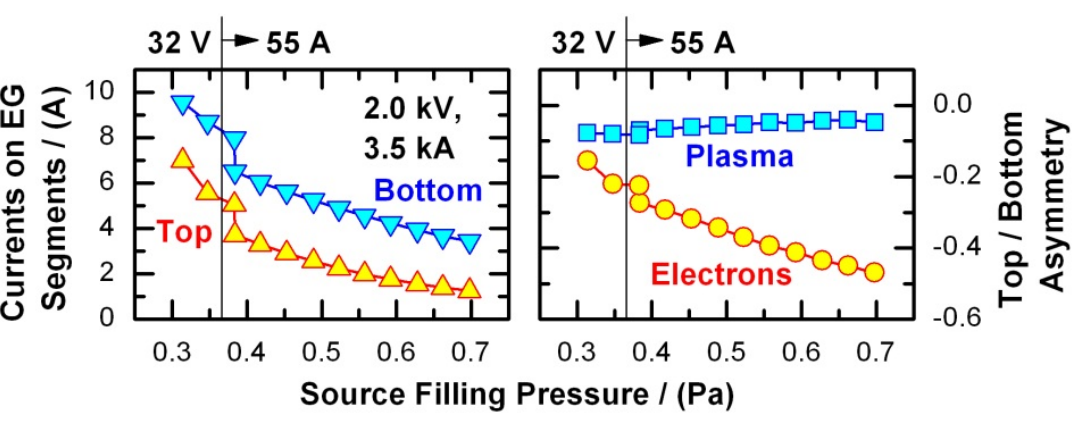

Figure 10: Dependence of the extracted currents and their symmetry on the source filling pressure in volume operation, i.e. w/o Cs. RF power was $2 \times 40 \mathrm{~kW}$; bias current $55 \mathrm{~A}$ or bias voltage $32 \mathrm{~V}$ as indicated, $P G$ filter field current $3.5 \mathrm{kA}$.

when changing bias and

filter field currents is in clear contrast to the findings at the small prototype source, where a large vertical asymmetry (corresponding to $|A| \approx 1$ ) was observed also in the Balmer lines $[20,32]$.

Finally, Figure 10 shows the dependence of the extracted electron currents on the source filling pressure. As already known from the small prototype source the co-extracted electron currents increase in volume operation with decreasing source filling pressure [12]. The asymmetry of the electron extraction, however, decreases with decreasing filling pressure, whereas the plasma asymmetry is again rather low and almost independent on the source pressure.

In summary, two main observations can be made by this new measurement of the individual electron currents at the extraction grid segments:

- The asymmetry of the extracted electron currents is not correlated with the asymmetry of the main plasma upstream of the bias plate. In fact, the plasma asymmetry is almost constant for all the parameters that have been changed during this study, with only one exception, when the filter field is rather low (below about $1 \mathrm{kA}$, see Figure 9). In these conditions, however, extraction is not possible.

- Generally, the asymmetry increases with increasing bias current/voltage (above a certain value, i.e. $20 \mathrm{~A}$ bias current), with increasing filter field and with decreasing extraction voltage. In parallel, the currents itself are decreasing. The asymmetry, however, is correlated with the amount of the currents: in all examples shown here, the asymmetry decreases with increasing extracted currents (see Figure 11), indicating some current offset effect. The same behavior is however seen in the calorimetric measurements (not shown here); hence this offset is not an offset in the current measurement itself.

The reason and the detailed processes leading to the observed asymmetry of the electron extraction are an open issue. The bias current/voltage has the most significant 


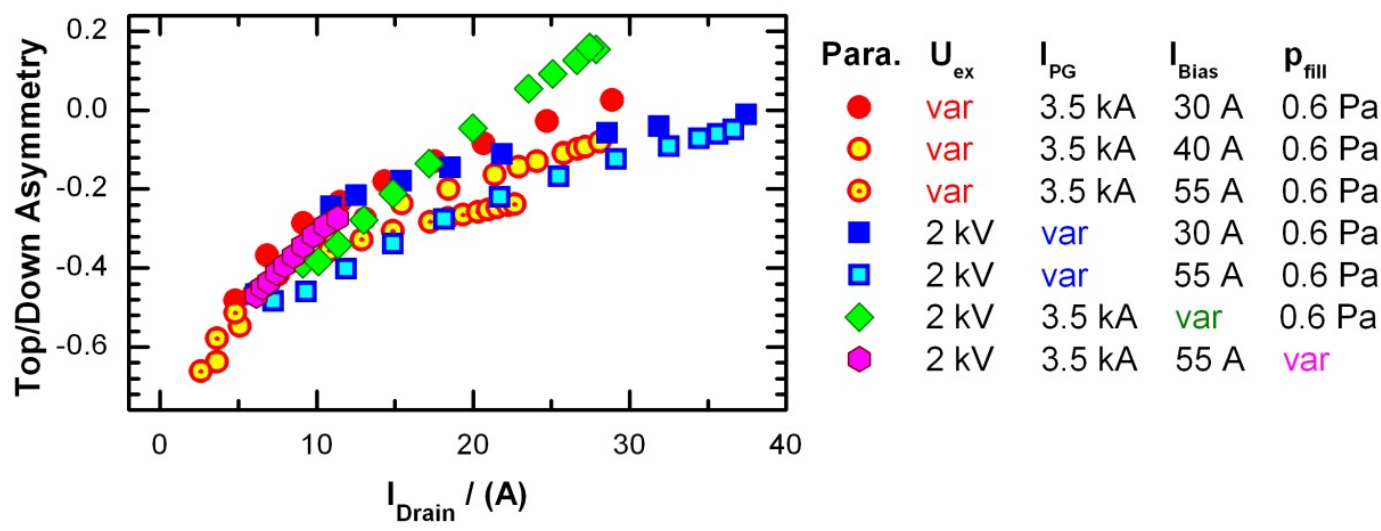

Figure 11: Dependence of the asymmetry of the extracted electron currents on the drain current, for all the parameter variations shown in the figures above.

effect on the asymmetry,, which indicates processes, i.e. drifts, in the extraction region just before the plasma grid. For bias currents $<20 \mathrm{~A}$ the asymmetry points also in the right direction for plasma drifts (upwards), but the 'drift' reversion for large bias currents is rather puzzling. For these high bias currents the electrons are deflected downwards as it is the case for the ions (as seen on the calorimeter, not shown here), so that a deflection of the electrons in the fringe field of the magnetic filter field in the first gap may explain the asymmetry. This explanation is supported by the dependence of the asymmetry on the filter field and the extraction voltage; but the amount of the asymmetry and the long necessary distances of the electron trajectories from one EG segment to the other are hard to explain with the present understanding and models of electron extraction: electron deflection codes like TRAJANe - a version of the IPP TRAJAN code [33] for electron extraction and EG power loads - show that a change of the magnetic filter fringe field in the extraction gap just rotates the local power deposition profile around the aperture [16], i.e. the electrons do not 'leave' their birth aperture.

In order to tackle the lack of understanding of the electron transport in the extraction region upstream the plasma grid and in the extraction gap downstream the plasma gap, experiments have been performed where only one pair of drivers, i.e. the upper or the lower one, respectively, was powered. The results are discussed in the next section.

\subsection{Asymmetry for Operation with One Generator Only}

Figure 12 shows the dependence of the currents on the extraction grid segments on the magnetic filter field current and on the bias current, respectively, for the case when only one generator, i.e. only one pair of drivers is powered. The first main result is that the source behaves rather symmetrically: the extracted currents on the segments, which correspond to the powered pair of drivers, i.e. top segment for generator 1 and bottom segment for generator 2, are almost identical. A similar reduction of the currents with increasing bias current and filter field current can be seen as it was the case when both pairs of drivers are powered. 
Some current, however, can be also measured on the extraction grid segments, which are opposite to the powered pair of driver, i.e. on the bottom segment, when the upper pair of drivers (G1) is powered, and on the top segment, when the lower pair of drivers (G2) is powered. These currents show also a strong asymmetry: the current of the top segment is, when G2 (bottom) is powered, in most of the cases much larger than the current on the bottom segment when G1 (top) is powered. These small currents may be the cause of the above mentioned current offset for the operation with two pairs of driver leading to an increase of the asymmetry with decreasing absolute currents.

The asymmetry of top and bottom extraction grid current is shown in Figure 13 and compared with the plasma asymmetry.
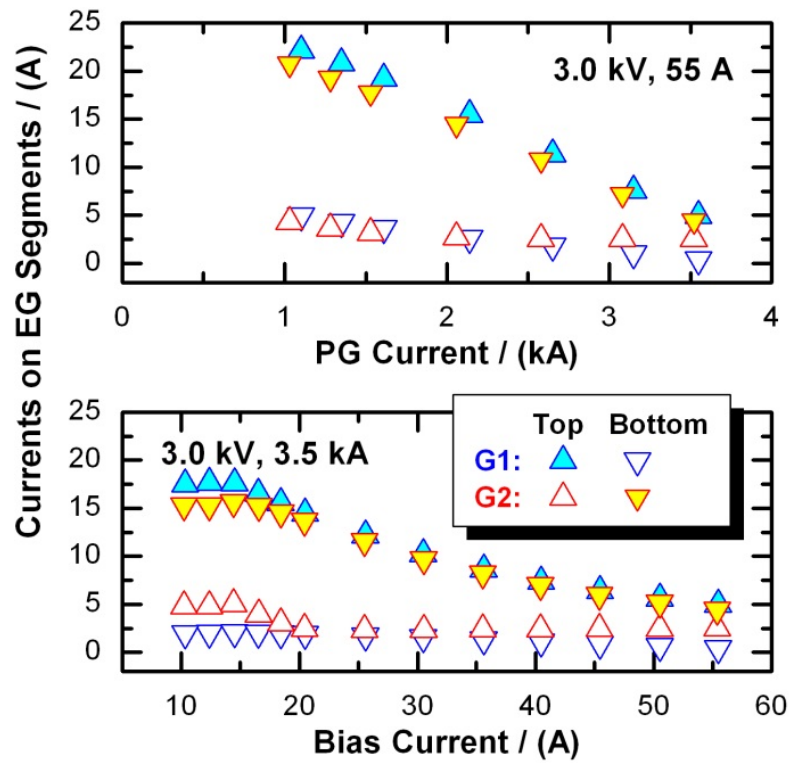

Figure 12: Dependence of the extracted electron currents on the magnetic filter field current (top) and the bias current (bottom) in volume operation, i.e. w/o Cs. Only one generator was powered, RF power was $40 \mathrm{~kW}$. The open points indicate currents on the opposite EG segment. G1: upper row of drivers; G2: lower row of drivers.

The latter is - as it was the case for the operation with two generators - more or less independent on the magnetic filter field or on the bias current; it is at around \pm 0.8 , where the sign depends as expected on the location of the powered pair of drivers. The asymmetry of the extracted electron currents, however, shows clear trends, but the direction depends on which pair of drivers is powered: the asymmetry increases slightly with increasing magnetic filter field and increasing bias current above $20 \mathrm{~A}$ for the case when the upper pair of drivers is powered, whereas it decreases when the lower pair is powered. In both cases, however, the asymmetry changes so that the electrons tend to go upwards towards the upper segment of the extraction grid, i.e. the current on the upper segment increases. This increase is in clear agreement with the electron drift direction for the present magnetic filter field direction, indicating that an ExB drift may cause the observed asymmetry.

In order to test this hypothesis, the data of Figure 12 and Figure 13 are plotted versus a drift parameter $U_{\text {bias }} \times I_{P G}$, i.e. the product of the plasma grid bias voltage and the plasma grid current. The plasma grid current is proportional to the magnetic filter field in front of the plasma grid. The bias potential is in principle proportional to the electric fields in front of the plasma grid when the plasma potential is not changing. Unfortunately, plasma potential measurements could be not performed up to now as a Langmuir probe system was not operational yet. This drift parameter must not be mistaken with the ExB drift velocity which depends on $E \times B / B^{2}$; measured here is the final result of a drift offset. The results 
are presented in Figure 14, where the dependence of the currents on both grid segments on this drift parameter is shown, and Figure 15 where the dependence of the resulting asymmetry is shown.

The results are surprising: for both parameter variations, bias current and magnetic filter field, the currents on both segments and their asymmetry can be sorted very well by the drift parameter, i.e. they are aligned on the same curve. This indicates indeed that an ExB drift is one of the drivers of the observed asymmetries of the electron extraction.

\subsection{Discussion}

The first results on the electron extraction at ELISE reported in this paper are very promising for the operation of a large RF driven ITER-relevant ion source, although there are done in volume operation, i.e. where the negative hydrogen ions are generated in the plasma volume

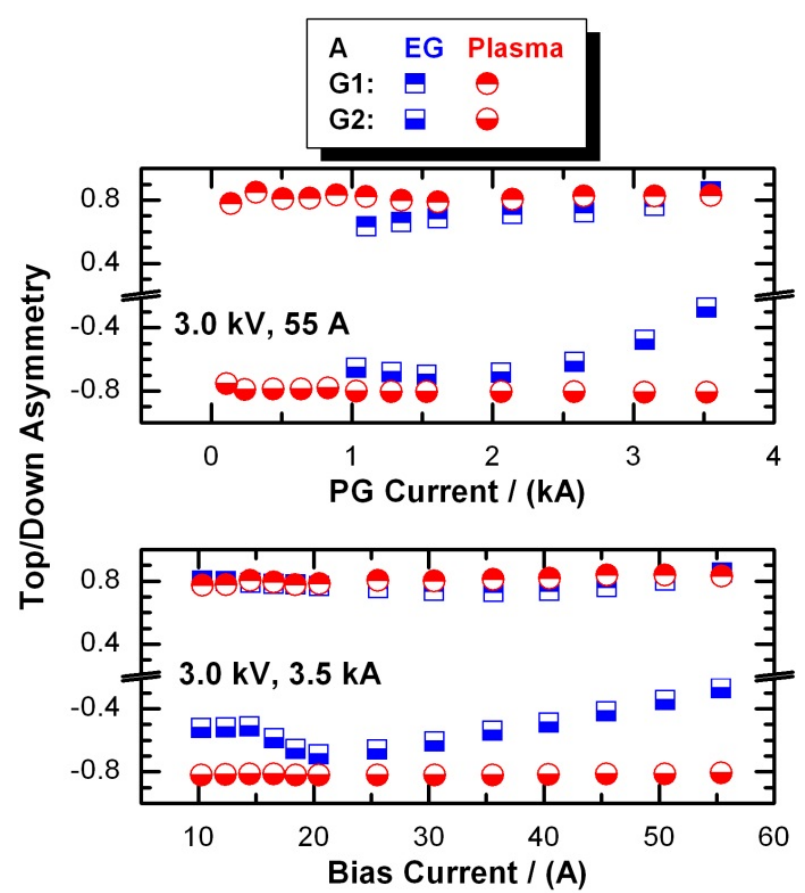

Figure 13: Dependence of the asymmetry $A$ of the extracted electron currents ('EG') and of the plasma on the magnetic filter field current (top) and the bias current (bottom) in volume operation, i.e. w/o Cs. Only one generator was powered (indicated by the half-filled points), RF power was $40 \mathrm{~kW}$. G1: upper row of drivers; G2: lower row of drivers.

solely. The most important point is that the amount of co-extracted electrons can be very efficiently suppressed with plasma grid bias and magnetic filter field. The electron suppression seems to be much more effective than in the small IPP prototype source: although there are not much data on electron extraction for volume operation, the maximum extraction voltage was mostly one or two $\mathrm{kV}$ at maximum in order to protect the extraction grid. This effective electron suppression in volume operation in ELISE gives some confidence that the amount of electrons can be kept well below the ion current as it is required - also for larger RF powers and deuterium - for the operation with Cs, where an additional electron suppression mechanism is present, i.e. the substitution of the electrons by negative hydrogen ions generated near the plasma grid apertures.

The most striking feature is the observed asymmetry of the co-extracted electron currents, which can be measured at ELISE for the first time with some spatial resolution, i.e. at the upper and lower extraction grid segment. This asymmetry of electron extraction is not correlated at all with the plasma asymmetry upstream of the bias plate - which is anyway small for the ITER relevant operational regime, in contrast to the small IPP prototype source 
- indicating that the region around the meniscus at the plasma grid apertures is decoupled from the main bulk plasma region upstream of the bias plate.

Both effects, the effective electron suppression and the decoupling of the region near the meniscus from the main plasma, are most probably a consequence of the main differences of the ELISE source to the small prototype source with respect to geometry and filter field configuration: the size of the source is much larger and, perhaps even more important, the bias plate geometry with the stripes also in the center of the source leading to areas there where the plasma sees no bias. Additionally, the transport of the electrons towards the source walls might be enhanced at ELISE due to the lack of a magnetic filter field mirror there leading to a reduced electron density in the extraction region.

The reason for the observed asymmetry of the electron extraction and their dependence on the operational parameters like extraction voltage, plasma grid bias and magnetic filter field is presently not quite well understood. Most probably, a drift of the electrons in the region just near the plasma meniscus is the main driver, and not the deflection of electrons in the fringe field within the extraction system. This is indicated by the experiments when only one pair of drivers was powered: the data could be sorted quite well by the product of bias voltage and magnetic filter field current, indicating an ExB drift. The change of asymmetry was also much more pronounced for powering the lower drivers, i.e. for the case, when there is space for the plasma drift which is upwards for the present magnetic filter field configuration. In the case of powering the upper drivers,

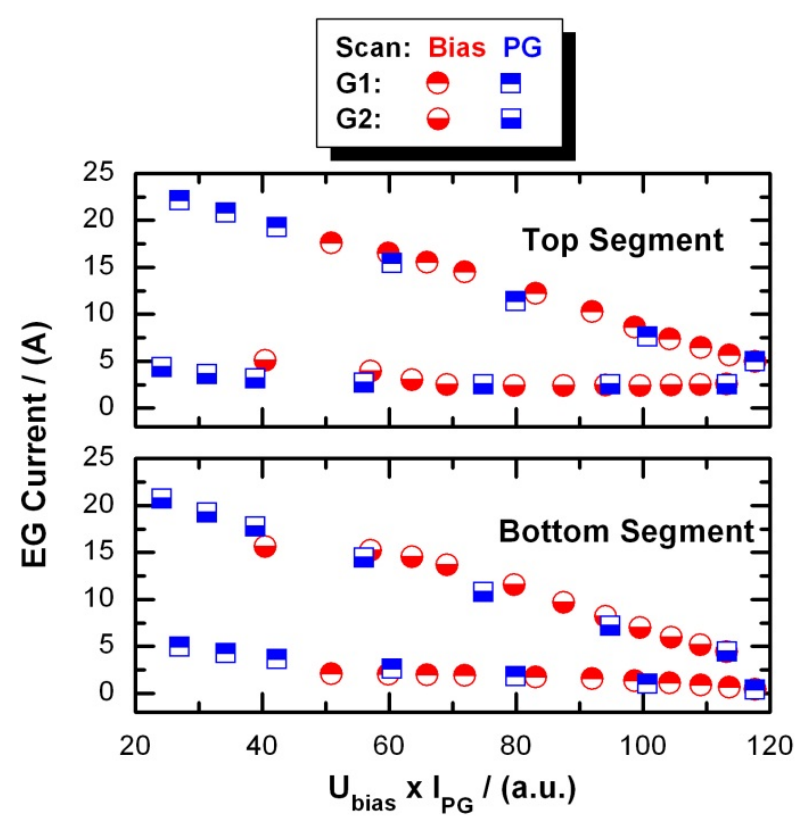

Figure 14: Dependence of the extracted electron currents on both segments for the two scans shown in Figure 12 on the product of bias voltage and filter field current. G1: upper row of drivers; G2: lower row of drivers.

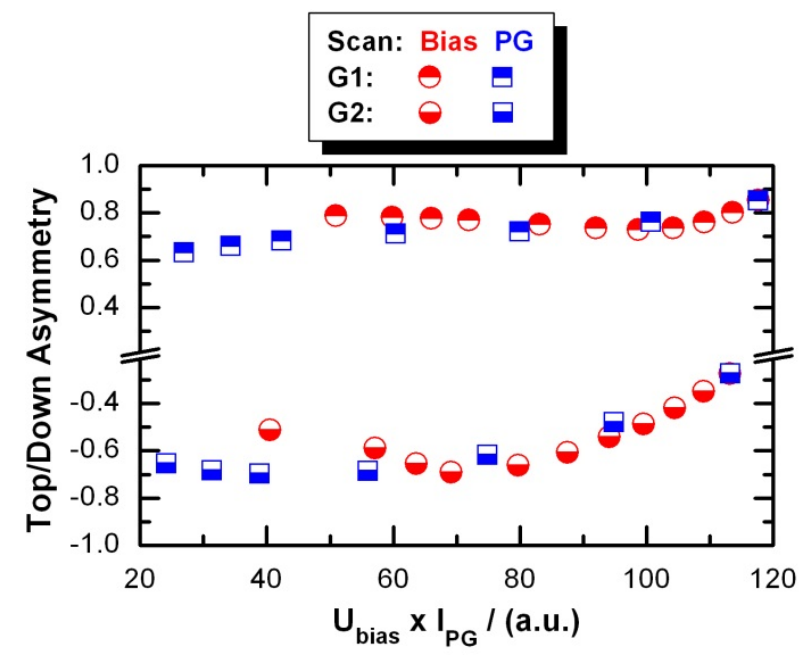

Figure 15: Dependence of the asymmetry of the extracted electron currents for the two scans shown in Figure 12 on the product of bias voltage and filter field current. G1: upper row of drivers; G2: lower row of drivers. 


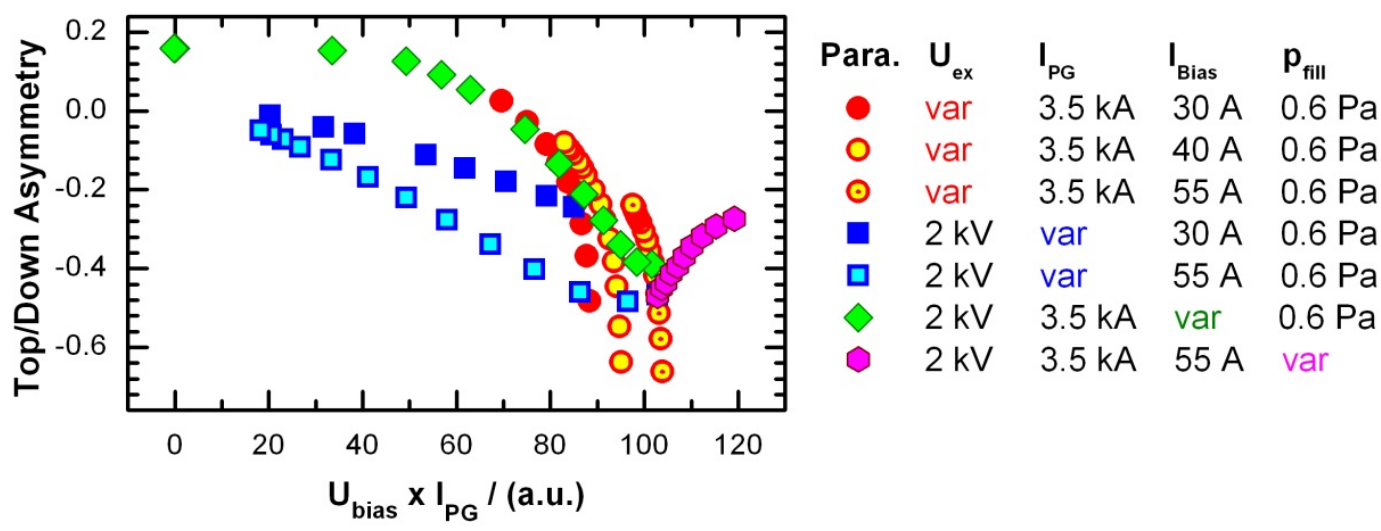

Figure 16: Dependence of the asymmetry of the extracted electron currents on the product of bias voltage and filter field current for all the parameter variations shown in the figures in section 3.2 with all four drivers powered.

the source wall hinders then apparently the drift.

There is however one drop of bitterness: the 'drift' direction in the case of the operation with both pairs of drivers where the current asymmetry is getting more and more negative, i.e. from $A \approx 0$ to $A \approx-0.4$ or -0.5 , is opposite to the case of the operation with one pair of drivers only where the current asymmetry is getting more and more positive, i.e. from $A \approx-0.7$ to $A \approx-0.3$ when the lower pair of drivers is powered, and from $A \approx+0.7$ to $A \approx+0.8$, when the upper pair of drivers is powered, respectively. This different behaviour is again highlighted in Figure 16, where the asymmetry of the electron extraction for the case of four driver operation is shown in dependence on the drift parameter bias voltage times magnetic filter field current. In contrast to the operation with only one pair of drivers, this parameter does not sort the observed asymmetry very well, even for the cases where just the bias and the filter field are varied. There is even one exemption from the common trend of increasing asymmetry with increasing bias and filter field: when the bias voltage is changed due to a change of the source pressure, the asymmetry decreases. This indicates that other processes are also contributing to the electron extraction asymmetry when the pressure is changing, maybe a different pattern of the plasma flow out of the drivers as observed at the IPP large ion source test facility RADI [14].

All these findings indicate that edge effects caused by the source walls as well as the mutual influence of the plasma from both pairs of drivers are interacting in a very complex manner in front of the plasma grid. The upcoming commissioning of a Langmuir probe system will give the possibility to correlate the findings above with measurements of the electron density and temperature and of the plasma potential. Unfortunately, similar experiments with a large range of operational parameters are not possible in deuterium where the plasma asymmetry is much less than in hydrogen [20]; due to the much larger coextracted electron current the power on the extraction grid exceeds in most of the cases the power limit. Anyway, in order to have more insight in this important physics of electron 
transport in large RF driven ion sources, full large scale 3D models of the ELISE source are highly desirable.

\section{Conclusion}

First measurements of the dependence of the co-extracted electron currents on various operational parameters at the large ITER-relevant source of the ELISE test facility have been performed. ELISE has the unique feature, that the electron currents can be measured individually on both extraction grid segments, leading to some vertical spatial resolution. The results, although obtained in volume operation, are very encouraging for the upcoming Cs phase and the ITER NBI injectors: The amount of co-extracted electrons could be suppressed very sufficiently with moderate magnetic filter field and by plasma grid bias. Furthermore, the electron extraction is more or less decoupled from the plasma upstream the bias plate, as the observed vertical asymmetry of electron extraction is not correlated at all with the main bulk plasma asymmetry, which is anyway rather small. Both effects are superior to the experience from the small IPP prototype source; the reason for these encouraging results are most probably the larger size of the source, the new geometry of the bias plate having also unbiased areas in the centre of the source and the different magnetic filter field topology.

The asymmetry of the electron extraction, however, is not very well understood. Experiments with only one generator, i.e. powering only half of the source, indicate that, if there is space for the plasma to drift, the electron extraction is mainly driven by ExB drifts near the extraction apertures. The situation is not so clear when the plasma drift is hindered by the source walls. More detailed full 3D large scale models are desirable for a better understanding.

\section{Acknowledgments}

The work was supported by a contract from Fusion for Energy (F4E-2009-0PE-32-01), represented by Antonio Masiello, with an amount of $4 \mathrm{M} €$. The opinions expressed herein are those of the authors only and do not represent the Fusion for Energy's official position.

\section{References}

[1] HEMSWORTH, R. et al. Nuclear Fusion, v. 49, p. 045006, 2009.

URL: <doi:10.1088/0029-5515/49/4/045006>.

[2] SCHUNKE, B. et al. AIP Conference Proceedings, v. 1097, p. 480, 2008.

URL: <doi:10.1063/1.3112548>.

[3] MASIELLO, A. et al. Fusion Engineering and Design, v. 84(7-11), p. 1276-1280, June 2009. URL: <http://dx.doi.org/10.1016/j.fusengdes.2008.12.090>. 
[4] MASIELLO, A. et al. Proceedings of the 24th IAEA Fusion Energy Conference. 2012. p. ITR/P1-04.

URL: <http://wwwnaweb.iaea.org/napc/physics/FEC/FEC2012/papers/361_ITRP104.pdf>.

[5] FRANZEN, P. et al. Fusion Engineering and Design, 2013. in press.

[6] NOCENTINI, R. et al. 25th Symposium on Fusion Engineering (SOFE 25). 2013. p. PID2814993.

[7] SONATO, P. et al. Proceedings of the 23rd IAEA Fusion Energy Conference. 2010. p. ITR/P1-13.

URL: <http://www-

pub.iaea.org/mtcd/meetings/PDFplus/2010/cn180/cn180_papers/itr_p1-13.pdf>.

[8] SCHUNKE, B. et al. Proceedings of the 24th IAEA Fusion Energy Conference. 2012. p. ITR/P1-03.

URL: <http://wwwnaweb.iaea.org/napc/physics/FEC/FEC2012/papers/227_ITRP103.pdf>.

[9] ROTTI, C. et al. Proceedings of the 24th IAEA Fusion Energy Conference. 2012. p. ITR/P543.

URL: <http://wwwnaweb.iaea.org/napc/physics/FEC/FEC2012/papers/599_ITRP543.pdf>.

[10] SONATO, P. et al. Proceedings of the 24th IAEA Fusion Energy Conference. San Diego, USA: IAEA. 2012. p. ITR/1-3.

URL: <http://wwwnaweb.iaea.org/napc/physics/FEC/FEC2012/papers/523_ITR13.pdf>.

[11] SONATO, P. et al. Fusion Engineering and Design, v. 84, n. 2-6, p. 269-274, 2009.

URL: <http://dx.doi.org/10.1016/j.fusengdes.2008.11.095>.

[12] SPETH, E. et al. Nuclear Fusion, v. 46, p. S220, 2006.

URL: <http://www.iop.org/EJ/abstract/0029-5515/46/6/S03>.

[13] FRANZEN, P. et al. AIP Conference Proceedings, v. 1097, p. 451, 2009.

URL: <http://link.aip.org/link/?APCPCS/1097/451/1>.

[14] FANTZ, U. et al. Nuclear Fusion, v. 49, p. 125007, 2009. URL: <doi:10.1088/0029-5515/49/12/125007>.

[15] FANTZ, U.; SCHIESKO, L.; WÜNDERLICH, D. AIP Conference Proceedings, v. 1515, p. 187, 2013.

URL: <http://dx.doi.org/10.1063/1.4792785>. 
[16] NOCENTINI, R. et al. Fusion Engineering and Design, v. 86(6-8), p. 916-919, 2011.

URL: <http://dx.doi.org/10.1016/j.fusengdes.2011.01.147>.

[17] FRANZEN, P. et al. Plasma Physics and Controlled Fusion, v. 53, p. 115006, 2011. URL: <http://dx.doi.org/10.1088/0741-3335/53/11/115006>.

[18] FRANZEN, P. et al. Nuclear Fusion, v. 51, p. 073035, 2011. URL: <http://dx.doi.org/10.1088/0029-5515/51/7/073035>.

[19] FANTZ, U.; FRANZEN, P.; WÜNDERLICH, D. Chemical Physics, v. 398, p. 7-16, 2012. URL: <doi:10.1016/j.chemphys.2011.05.006>.

[20] FANTZ, U. et al. AIP Conference Proceedings, v. 1097, p. 265, 2009. URL: <doi:10.1063/1.3112521>.

[21] SCHIESKO, L. et al. Plasma Phys. Control. Fusion, v. 54, p. 105002, 2012. URL: <doi:10.1088/0741-3335/54/10/105002>.

[22] WÜNDERLICH, D. et al. AIP Conference Proceedings, n. 1515, p. 12, 2013. URL: <http://dx.doi.org/10.1063/1.4792765.>.

[23] WÜNDERLICH, D. et al. Plasma Physics and Controlled Fusion, v. 54, p. 125002, 2012. URL: <http://dx.doi.org/10.1088/0741-3335/54/12/125002>.

[24] WÜNDERLICH, D. Special Issue of Plasma Physics and Controlled Fusion, 2013. to be published.

[25] HEINEMANN, B. et al. Fusion Engineering and Design, v. 84(2-6), p. 915-922, June 2009. URL: <http://dx.doi.org/10.1016/j.fusengdes.2008.11.076>.

[26] FRANZEN, P. et al. AIP Conference Proceedings, v. 993, p. 51-54, 2008. URL: <http://dx.doi.org/10.1063/1.2909175>.

[27] FRANZEN, P. et al. Fusion Engineering and Design, v. 82, p. 407-423, 2007. URL: <http://dx.doi.org/10.1016/j.fusengdes.2007.03.041>.

[28] FRÖSCHLE, M. et al. Fusion Engineering and Design, 2013. in press; availabe online.

[29] NOCENTINI, R. et al. Fusion Engineering and Design, v. 84, p. 2131-2135, 2009. URL: <doi:10.1016/j.fusengdes.2009.02.015>.

[30] WÜNDERLICH, D. et al. Review of Scientific Instruments, v. 84, p. 093102, 2013. URL: <http://link.aip.org/link/doi/10.1063/1.4820806>.

[31] FRANZEN, P. et al. Nuclear Fusion, v. 47, p. 264-270, 2007. URL: <doi:10.1088/0029-5515/47/4/004>.

[32] FRANZEN, P.; FANTZ, U.; NNBI-TEAM. AIP Conference Proceedings, v. 1390, p. 310-321, 2011.

URL: <http://dx.doi.org/10.1063/1.3637401>. 
[33] GUTSER, R. et al. Plasma Phys. Control. Fusion, n. 51, p. 045005, 2009. URL: <doi:10.1088/0741-3335/51/4/045005>. 